\title{
Modelo para as Interações entre Células de Defesa Contra o HIV
}

\section{Edilson F. Arruda ${ }^{1}$}

Instituto Alberto Luiz Coimbra de Pós Graduação e Pesquisa de Engenharia, Universidade Federal do Rio de Janeiro, Rio de Janeiro, RJ

\section{Claudia M. Dias ${ }^{2}$}

Instituto Multidisciplinar, UFRRJ, Av. Governador Roberto Silveira, Nova Iguaçu, RJ

\section{Dayse H. Pastore, ${ }^{3}$ Roberto Carlos A. Thomé ${ }^{4}$}

Departamento de Matemática, CEFET/RJ, Rio de Janeiro, RJ

Hyun M. Yang 5

Departamento de Matemática Aplicada, UNICAMP, Campinas, SP

Resumo. Nesse trabalho faremos uma análise da capacidade do organismo humano de enfrentar o HIV. O modelo aqui tratado leva em consideração quatro tipos de células de defesa de um organismo infectado pelo HIV: as células de defesa suscetíveis, as células de defesa infectadas, as células $\mathrm{T}$ assassinas, e as células $\mathrm{T}$ assassinas específicas para $\mathrm{o}$ vírus HIV. Esse modelo, portanto, analisa as interações entre as respostas das células T assassinas e as infecções causadas pelo HIV. Veremos como o sistema imunológico é atacado e como este se defende. Faremos também uma análise sobre o efeito de como as drogas usadas nos tratamentos contra essa doença aumentam a capacidade de defesa do organismo infectado.

Palavras-chave. HIV, Modelagem Matemática, Controle.

\section{Introdução}

Modelos matemáticos têm contribuído na compreensão da dinâmica de populações [3]. No início da década de 1990, modelos populacionais começaram a ser usados por um certo número de pessoas, a fim de descrever a dinâmica in vivo entre as infecções virais e as respostas imunológicas, em particular para o vírus da imunodeficiência humana (HIV) [6-8]. Na ocasião a ênfase era a questão viral destas dinâmicas, incluindo a estimativa de parâmetros básicos virais, a dinâmica evolutiva de fuga imune pelo vírus, e a análise do

\footnotetext{
${ }^{1}$ efarruda@po.coppe.ufrj.br

${ }^{2}$ mazzaclaudia@gmail.com

${ }^{3}$ dayse.pastore@cefet-rj.br

${ }^{4}$ roberto.thome@cefet-rj.br

${ }^{5}$ hyunyang@ime.unicamp.br
} 
tratamento da toxicodependência na infecção pelo HIV. Uma parte específica do sistema inumológico é formada pelas células T assassinas ou linfócitos T Citotóxicos (CTL). Essas células são muito importantes na luta contra as infecções virais.

Propomos uma variação do modelo apresentado em $[1,4]$. Esse modelo apresentou a introdução de uma nova variável que é chamada de células específicas de defesa ativadas. Nesse modelo os autores usaram o vírus livre do HIV no organismo como ativadores das células de defesa, assim tornando-as especificas para esse vírus. Aqui consideraremos que quem ativa essas células são as células infectadas. Essa modificação tem base em um novo estudo que eleva em consideração a interação entre as células infectadas e o organismo infectado.

\section{Apresentação do Modelo}

Quando o HIV invade o corpo humano, o alvo são as células de defesa T CD4+ presentes no organismo. Essas células, consideradas como "auxiliares", sinalizam a presença de um invasor para outras células de defesa (B e T CD8+). As células T CD8+ respondem a esse sinal partindo para a destruição das células infectadas. A partir dessa resposta elas se tornam específicas para o HIV. Neste trabalho, estamos propondo um novo modelo matemático para estudar a dinâmica do HIV no sistema imunológico humano, com base em vários modelos existentes na literatura $[2,4,9]$. Esse modelo é dado pelo sistema de equações diferenciais ordinárias que reproduziremos a seguir:

$$
\left\{\begin{array}{l}
\dot{x}=\lambda_{x}-\mu_{x} x-\beta_{v} x v-u_{1} x \\
\dot{x_{p}}=u_{1} x-\mu_{x} x_{p} \\
\dot{y}=\beta_{v} x v-\mu_{y} y-p_{y} y z_{a}-u_{2} y \\
\dot{y_{b}}=u_{1} y-\mu_{y} y_{b} \\
\dot{v}=k_{v} \mu_{y} y-\mu_{v} v \\
\dot{z}=\lambda_{z}-\mu_{z} z-\beta_{z} z y \\
\dot{z_{a}}=\beta_{z} z y-\mu_{z} z_{a}
\end{array}\right.
$$

Nas equações acima, a variável $x$ representa as células suscetíveis, i.e., as células as quais o HIV se conecta, a variável $x_{p}$ representa as células suscetíveis protegidas por inibidores de transcriptase reversa, protease e entrada, a variável y representa essa células após o HIV ter se conectado, já a variável $y_{b}$ representa as células bloqueadas por um inibidor de protease. Utilizamos $v$ para representar os vírus livres presentes no organismo, a variável $z$ é a população de todas as células $\mathrm{T}$ assassinas da resposta imunológica em repouso e a variável $z_{a}$ é a população dessas células ativadas no combate as células infectadas, que está respondendo com anticorpos.

Como condição inicial consideraremos o instante em que o vírus tem seu primeiro contato com o organismo, ou seja, consideraremos o ponto de equilíbrio trivial do sistema sem a presença do vírus e introduziremos uma pequena quantidade desse vírus: $x(0)=$ $\lambda_{x} / \mu_{x}, x_{p}(0)=0, y(0)=0, y_{b}(0)=0, v(0)=v_{0}, z(0)=\lambda_{z} / \mu_{z}, z_{a}(0)=0$.

Nas equações $u_{1}$ e $u_{2}$ são as variáveis de controle da medicação. Observe que no caso onde $u_{1}=u_{2}=0$, as variáveis $x_{p}$ e $y_{b}$ se anulam sempre. Ou seja, neste caso podemos 


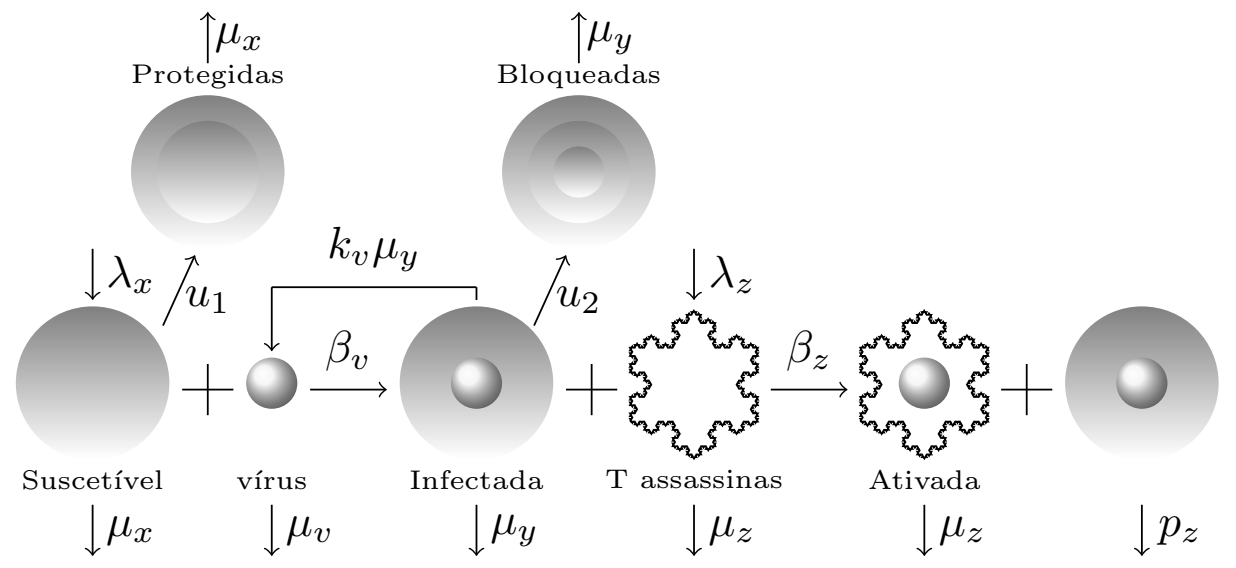

Figura 1: Dinâmica do Sistema

considerar que não há controle. Ou ainda, o paciente não foi exposto a nenhum tipo de tratamento.

O mecanismo da dinâmica do sistema sem controle está descrito na Figura 1, observe que os vírus $(v)$ e as células suscetíveis $(x)$ produzem células infectadas $(y)$ que por sua vez produzem o vírus $(v)$. Note que as células assassinas $(z)$ são ativadas pelas células infectadas $(y)$ e essas por sua vez eliminam as células infectadas $(y)$ que as ativaram.

Os parâmetros usados no sistema de equações estão descritos na Tabela 1. Consideramos que as células suscetíveis $x$ são produzidas em uma quantidade constante $\lambda_{x}$, e decaem a uma taxa também constante $\mu_{x}$. Além disso, consideramos que as células suscetíveis são infectadas pelo vírus a uma taxa $\beta_{v}$. Sendo assim, a variável $x$ muda para o compartimento da variável $y$ a uma taxa $\beta_{v}$. A variável $y$ decai a uma taxa $\mu_{y}$ e é eliminada do sistema a uma taxa $p_{y}$ que é a taxa de combate do organismo pelas células $\mathrm{T}$ assassinas ativadas para o HIV. O vírus livre $v$ é produzido a uma taxa $k_{v}$ (estamos considerando a morte da célula infectada, quando essa libera os vírus), a taxa de decaimento dessas células é $\mu_{v}$. As células $\mathrm{T}$ assassinas são produzidas a uma taxa constante $\lambda_{z}$, e decaem a uma taxa $\mu_{z}$. Consideramos ainda uma taxa de ativação dessas células quando essas interagem com uma célula infectada $\beta_{z}$, o que faz com que essas células migrem do compartimento de células assassinas para o compartimento de células assassinas ativadas. Por fim as células ativadas decaem com a mesma taxa das não ativadas.

A variável de controle $u_{1}$ irá representar o efeito dos inibidores de transcriptase reversa, de integrase e de entrada. A ação desse medicamento irá proteger as células alvo $x$ impedindo que elas se tornem células infectadas $y$. Por esse motivo, introduzimos no nosso modelo a variável $x_{p}$ representando as células protegidas devido a ação desses inibidores. Por outro lado, a variável de controle $u_{2}$ simula o efeito do inibidor de protease que irá atuar bloqueando as células infectadas $y$ evitando que elas liberem vírus no organismo.

O valores usados nas simulações numéricas (vide Tabelas 1 e 2 ), foram retirados dos artigos $[5,8,9]$. 
Tabela 1: Parâmetros

\begin{tabular}{|l|c|c|}
\hline Mortalidade das células suscetíveis & $\mu_{x}$ & $0.02 \mathrm{dia}^{-1}$ \\
\hline Mortalidade das células infectadas & $\mu_{y}$ & $0.24 \mathrm{dia}^{-1}$ \\
\hline Mortalidade do vírus & $\mu_{v}$ & $2.4 \mathrm{dia}^{-1}$ \\
\hline Mortalidade das células T assassinas & $\mu_{z}$ & $0.04 \mathrm{dia}^{-1}$ \\
\hline $\begin{array}{l}\text { Número médio de vírus livre liberados por } \\
\text { uma célula infectada }\end{array}$ & $k_{v}$ & 360 \\
\hline Taxa de ativação da resposta imunológica & $\beta_{z}$ & $5 \cdot 10^{-6} \mathrm{~mm}^{3} \mathrm{dia}^{-1}$ \\
\hline Taxa de infecção do vírus & $\beta_{v}$ & $2.4 \cdot 10^{-5} \mathrm{~mm}^{3} \mathrm{dia}^{-1}$ \\
\hline Taxa de destruição de células infectadas & $p_{y}$ & $0.02 \mathrm{~mm}^{3} \mathrm{dia}^{-1}$ \\
\hline Taxa de suprimento das células suscetíveis & $\lambda_{x}$ & $20 \mathrm{dia}^{-1} \mathrm{~mm}^{-3}$ \\
\hline Taxa de suprimento das células T assassinas & $\lambda_{z}$ & $20 \mathrm{dia}^{-1} \mathrm{~mm}^{-3}$ \\
\hline $\begin{array}{l}\text { Controle através de inibidores de transcriptase } \\
\text { reversa, de integrase e de entrada }\end{array}$ & $u_{1}$ & {$[0,1]$} \\
\hline Controle através de inibidores de protease & $u_{2}$ & {$[0,1]$} \\
\hline
\end{tabular}

Tabela 2: Condições Iniciais

\begin{tabular}{|c|c|c|}
\hline Células suscetíveis & $x$ & $10^{3} \mathrm{~mm}^{-3}$ \\
\hline Células protegidas & $x_{p}$ & $0 \mathrm{~mm}^{-3}$ \\
\hline Células infectadas pelo HIV & $y$ & $0 \mathrm{~mm}^{-3}$ \\
\hline Células bloqueadas & $y_{b}$ & $0 \mathrm{~mm}^{-3}$ \\
\hline HIV livres no organismo & $v$ & $10^{-3} \mathrm{~mm}^{-3}$ \\
\hline Células T assassinas ativadas para o HIV & $z$ & $500 \mathrm{~mm}^{-3}$ \\
\hline Células de defesa ativadas & $z_{a}$ & $0 \mathrm{~mm}^{-3}$ \\
\hline
\end{tabular}

\subsection{Pontos de Equilíbrio}

Os pontos de equilíbrio do sistema dinâmico (1) são dados pela relação:

$$
\begin{aligned}
P= & \left(\bar{x}, \bar{x}_{p}, \bar{y}, \bar{y}_{b}, \bar{v}, \bar{z}, \bar{z}_{a}\right) \\
= & \left(\frac{\lambda_{x}}{\mu_{x}+u_{1}+\beta_{v} \bar{v}}, \frac{\lambda_{x} \mu_{x}}{u_{1}\left(\mu_{x}+u_{1}+\beta_{v} \bar{v}\right)}, \frac{\mu_{v} \bar{v}}{k_{v} \mu_{y}}, \frac{\mu_{v} \bar{v}}{k_{v} u_{2}}, \bar{v}, \frac{k_{v} \lambda_{z} \mu_{y}}{\beta_{z} \mu_{v} \bar{v}+k_{v} \mu_{y} \mu_{z}},\right. \\
& \left.\frac{\lambda_{z} \beta_{z} \mu_{v} \bar{v}}{\mu_{z}\left(\beta_{z} \mu_{v} \bar{v}+k_{v} \mu_{y} \mu_{z}\right)}\right)
\end{aligned}
$$

onde:

$$
\bar{y}=0 \quad \text { ou } \quad a \bar{y}^{2}+b \bar{y}+c=0,
$$

com

$$
\begin{aligned}
a= & \beta_{v} \beta_{z} \mu_{v}^{2}\left(\mu_{y} \mu_{z}+\lambda_{z} p_{y}+\mu_{z} u_{2}\right), \\
b= & \mu_{v}\left(\beta_{v} k_{v} \mu_{y}^{2} \mu_{z}^{2}+\beta_{z} \mu_{v} \mu_{x} \mu_{y} \mu_{z}+\beta_{z} \lambda_{z} \mu_{v} \mu_{x} p_{y}+\beta_{z} \mu_{v} \mu_{x} \mu_{z} u_{2}+\beta_{z} \mu_{v} \mu_{y} \mu_{z} u_{1}\right. \\
& \left.+\beta_{z} \lambda_{z} \mu_{v} p_{y} u_{1}+\beta_{z} \mu_{v} \mu_{z} u_{1} u_{2}+\beta_{v} k_{v} \mu_{y} \mu_{z}^{2} u_{2}-\beta_{v} \beta_{z} k_{v} \lambda_{x} \mu_{y} \mu_{z}\right), \\
c= & k_{v} \mu_{v} \mu_{x} \mu_{y}^{2} \mu_{z}^{2}+k_{v} \mu_{v} \mu_{y}^{2} \mu_{z}^{2} u_{1}-\beta_{v} k_{v}^{2} \lambda_{x} \mu_{y}^{2} \mu_{z}^{2}+k_{v} \mu_{v} \mu_{x} \mu_{y} \mu_{z}^{2} u_{2}+k_{v} \mu_{v} \mu_{y} \mu_{z}^{2} u_{1} u_{2} .
\end{aligned}
$$


Para uma pessoa não-infectada pelo HIV temos $\bar{v}=0$. Substituindo esse valor na expressão (2), temos o ponto de equilíbrio trivial:

$$
P_{o}=\left(\bar{x}, \bar{x}_{p}, \bar{y}, \bar{y}_{b}, \bar{v}, \bar{z}, \bar{z}_{a}\right)=\left(\frac{\lambda_{x}}{\mu_{x}+u_{1}}, \frac{\lambda_{x} \mu_{x}}{u_{1}\left(\mu_{x}+u_{1}\right)}, 0,0,0, \frac{\lambda_{z}}{\mu_{z}}, 0\right) .
$$

Já para uma pessoa infectada pelo HIV, temos $\bar{v} \neq 0$. Com isso, temos que resolver a equação quadrática dada em (3). Vamos lembrar que um ponto de equilíbrio é estável, se o sinal da parte real dos autovalores da matriz jacobiana aplicada no ponto, a ser estudado, é negativa [teorema de Hartman-Grobman (Kreyszig, 1978)]. É fácil ver que a condição para que o ponto de estabilidade trivial desse sistema seja estável é:

$$
\frac{k_{v} \beta_{v} \lambda_{x} \mu_{y}}{\left(\mu_{x}+u_{1}\right)\left(\mu_{y}+u_{2}\right) \mu_{v}}<1 .
$$

Dessa forma, podemos concluir que o número de reprodução básica do vírus é dado por:

$$
R_{0}=\frac{k_{v} \beta_{v} \lambda_{x} \mu_{y}}{\left(\mu_{x}+u_{1}\right)\left(\mu_{y}+u_{2}\right) \mu_{v}} .
$$

Assim, se $R_{0}<1$, pelo que acabamos de ver em (5), o ponto de equilíbrio trivial $P_{o}$ dado em (4) é estável. Ou seja, nesse caso, a infecção não se propagaria no organismo do indivíduo.

\section{Simulações Numéricas e Conclusões}

Para realizar as simulações foi utilizado o método de Runge-Kutta (pacote ODE45 do software MatLab ${ }^{\circledR}$ ). Primeiramente simulamos o sistema com o tempo variando de 0 a 100 dias. Nessa primeira etapa da simulação utilizamos $u_{1}=u_{2}=0$, essa simulação do sistema nos fornece as condições de um paciente infectado pelo HIV a 100 dias sem tratamento. Finalmente, a partir das condições iniciais obtidas, simulamos o problema com tempo variando de 100 a 300 dias e variando os parâmetros $u_{1}$ e $u_{2}$, ver Figura 3. Desse modo, podemos ver o que acontece com o organismo quando ele é submetido a um tratamento, e com quantidades diferentes de drogas. Vemos que para um indivíduo sem tratamento a curva tenderá a se aproximar de um dos pontos de equilíbrio não-triviais do sistema (representados no gráfico por uma reta constante). Enquanto em um indivíduo tratado, a partir do centésimo dia se percebe a diminuição considerável do nível de carga viral no seu organismo e, consequentemente, a diminuição do nível de células infectadas. Os resultados se encontram na Figura 3. Podemos ainda observar que a quantidade de células suscetíveis ao HIV (a soma das células suscetíveis e das células suscetíveis bloqueadas) ao final de 300 dias se aproxima do valor encontrado em um organismo saudável. Ou seja, o tratamento consegue elevar o número de linfócitos T-CD4+ no organismo, ver Figura 2. Vemos também que a quantidade de células infectadas decai para zero, mesmo levando em consideração as células infectadas bloqueadas, ver Figura 2.

Em trabalhos futuros pretendemos aplicar a teoria de controle ótimo utilizando como variáveis de controle $u_{1}$ e $u_{2}$. Dessa forma podemos sugerir o melhor custo benefício para a utilização das terapias retro-virais. 

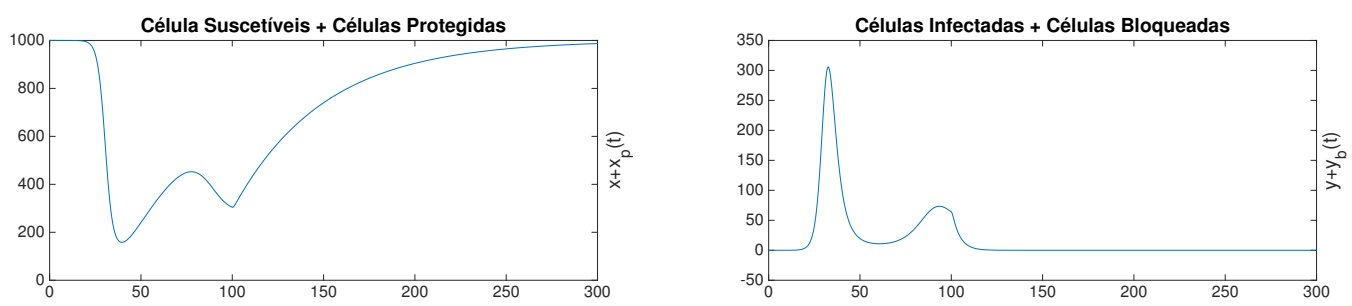

Figura 2: Resultados com Tratamento
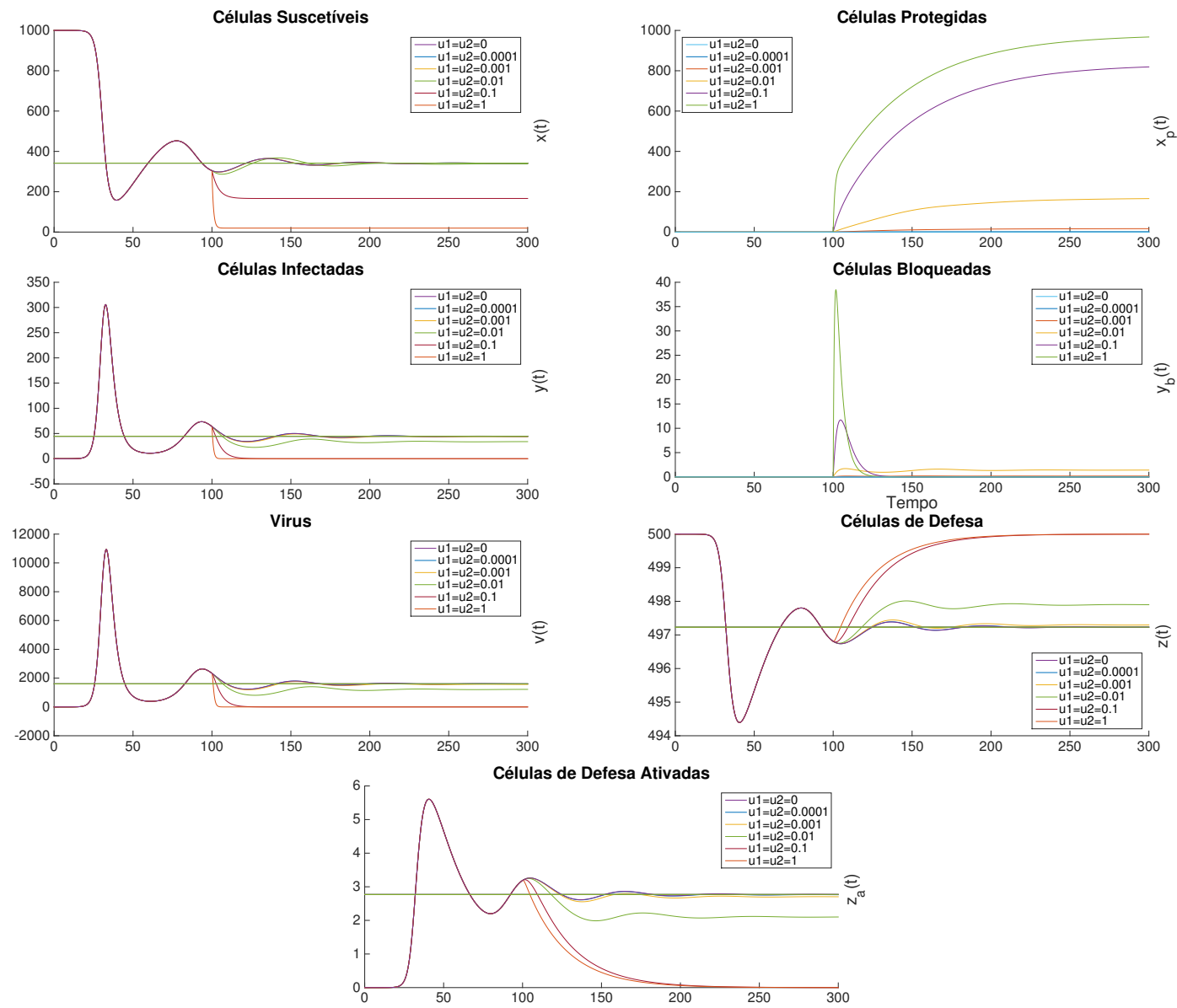

Figura 3: Simulação Numérica do Modelo

\section{Agradecimentos}

Oa autores agradecem a FAPERJ, pelo apoio financeiro fornecido pelos processos E26/110.379/2014 e E-26/110.087/2014. Este trabalho foi parcialmente financiado pela CAPES, processo de No. BEX2025/14-0, e pelo CNPq, através do processo No.302716/2011-4. 


\section{Referências}

[1] E. Arruda, C. Dias, C. de Magalhães, D. Pastore, R. Thomé and H. Yang, An Optimal Control Approach to HIV Immunology, Applied Mathematics, vol. 6, 1115-1130, (2015), DOI: 10.4236/am.2015.66102.

[2] J. Grégio, M. Caetano and T. Yoneyama, T., State estimation and optimal long period clinical treatment of HIV seropositive patients, Anais da Academia Brasileira de Ciências, vol. 81, 3-12, (2009).

[3] S.A. Levin, B. Grenfell, A. Hastings and A.S. Perelson, Mathematical and computational challenges in population biology and ecosystems science, Science, vol. 275, 334-343, (1997).

[4] C. Magalhães, R. Thomé, D.H. Pastore e H.M. Yang, Modelagem matemática da imunologia de HIV: O estudo das células de defesa ativadas, Tecnologia \& Cultura, vol. 22, 42-48, (2013).

[5] A. Mclean, Infectious disease modeling, In P. Kanki and D. J. Grimes, editors, Infectious Diseases, Springer, New York, 99-115, (2013).

[6] M. Nowak and C. Bangham, Population dynamics of immune responses to persistent víruses, Science, vol. 272, 74-79, (1996).

[7] M. Nowak and R. May, Virus dynamics: Mathematical principles of immunology and virology, Oxford University Press, Oxford, (2000).

[8] A. Perelson and P. Nelson, Mathematical analysis of HIV-1 dynamics in vivo, SIAM Rev. 4, 3-44, (1999).

[9] D. Wodarz, Killer Cell Dynamics Mathematical and Computational Approaches to Immunology, Interdisciplinary Applied Mathematics, vol. 32, Springer, (2006). 